\title{
INTELLIGENT SWITCHING CONTROL OF NONLINEAR NON-MINIMUM PHASE RELATIVE DEGREE TWO SYSTEMS
}

\author{
Jörg Mareczek*, Simon Hecker*, Martin Buss ${ }^{\diamond}$ \\ * Institute of Automatic Control Engineering \\ Technische Universität München \\ e-mail: joerg.mareczek@ieee.org, simon.hecker@gmx.de \\ Fax:+49-89-289-28340 \\ $\diamond$ Control Systems Group \\ Technical University Berlin \\ e-mail:m.busseieee.org
}

Keywords: nonlinear control, switching, geometric, nonminimum phase, underactuated robot

\begin{abstract}
In this paper we extend earlier results on Invariance Control to the special class of nonlinear control input affine systems with relative degree two. A switching strategy for control parameters and an easy to calculate invariance region together form an intelligent switching Invariance Controller, achieving asymptotic stability for the controlled dynamics and positive invariance of a prescribed bounded state space region. Experiments with an underactuated robot show the applicability of the proposed theory.
\end{abstract}

\section{Introduction}

Many important control systems have more degrees of freedom than actuators. Some examples are the Vertical Take Off and Landing (VTOL) plane, motor vehicles, marine vessels, combustion systems, and underactuated robots. Amongst existing nonlinear control methods some are suitable to control systems with reduced relative degree, e.g. passivity based control [2, 14], backstepping [4], etc. Output regulation has been addressed by $[1,5]$. Discontinuous feedback control algorithms have been proposed by [3, 10]. The novel method Invariance Control, keeps a prescribed state space region positively invariant and guarantees in addition asymptotic stability of the controlled dynamics. Choosing such an invariance region in the right way enables a control designer to take pre-specified state space constraints into account, to achieve internal stability, and to guarantee robustness with respect to system parameter perturbations and control input limitations. Having discussed Invariance Control for a general class of control input affine nonlinear systems in earlier work [7, 8] we now focus on the special class of nonlinear control input affine systems with relative degree two for the following two reasons:

1. A lot of underactuated systems belong to the class of mechanical or mechatronic systems. In the case of a SISOsystem feedback linearization leads to a double integrator subsystem. Hence, in spite of considering a general $r$ time integrator subsystem it makes sense to focus on the special case $r=2$, i.e. on a double integrator subsystem.
2. In the case of a relative degree two system, the design process of Invariance Control can be simplified, compared with the general case.

The paper is organized as follows: The system class and the control design goal is defined in Section 2. Then the main result consisting of an Invariance Control design method is presented in Section 3. Finally we illustrate the proposed theory by an experiment with an underactuated robot in Section 4.

\section{System Class and Control Design Goal}

In this paper we consider nonlinear SISO-systems of the form

$$
\begin{aligned}
\dot{x}_{1} & =x_{2} \\
\dot{x}_{2} & =u \\
\dot{\boldsymbol{z}} & =\boldsymbol{f}(\boldsymbol{x}, \boldsymbol{z})+\boldsymbol{g}(\boldsymbol{x}, \boldsymbol{z}) u
\end{aligned}
$$

with the system internal state $z \in \mathbb{R}^{n-2}$ and the controlled state $\boldsymbol{x}=\left[\begin{array}{ll}x_{1} & x_{2}\end{array}\right]^{T}$, a drift and control input vector field $\boldsymbol{f}, \boldsymbol{g} \in \mathcal{C}^{1}$ with $\boldsymbol{f}, \boldsymbol{g}: \mathbb{R}^{n} \rightarrow \mathbb{R}^{n-2}$. The control input $u$ is assumed to be unlimited.

Note that this class of system usually results from a normal form feedback linearization; thus the considered system class covers many practically relevant nonlinear systems.

Assumption 1 There exists a differentiable, radially unbounded function $\Phi_{0}\left(x_{1}, \boldsymbol{z}\right)$ defining an open and connected state space region $\mathcal{G}_{0}=\left\{\left(x_{1}, \boldsymbol{z}\right) \mid \Phi_{0}\left(x_{1}, \boldsymbol{z}\right) \leq 0\right\}$ with boundary $\partial \mathcal{G}_{0}$ such that

$$
\frac{\partial \Phi_{0}\left(x_{1}, \boldsymbol{z}\right)}{\partial \boldsymbol{z}} \boldsymbol{f}(\boldsymbol{x}, \boldsymbol{z}) \leq 0 \quad \forall(\boldsymbol{x}, \boldsymbol{z}) \in \partial \mathcal{G}_{0} \cap\left\{x_{2}=0\right\} .
$$

This means that the zero-dynamics manifold of system (1)-(3) with respect to an output function $h=x_{1}=$ const, with $x_{1} \in\left[x_{1}(0) ; 0\right]$, is supposed to be at least locally stable in the sense of Lyapunov.

The control design goal is as follows: Given a state space region $\mathcal{G} \subseteq \mathbb{R}^{n}$, find a controller $u(\boldsymbol{x}, \boldsymbol{z})$ such that with $(\boldsymbol{x}(0), \boldsymbol{z}(0)) \in \mathcal{G}$ we obtain for the controlled system (1)-(3) asymptotic stability

$$
\lim _{t \rightarrow \infty} \boldsymbol{x}(t)=\mathbf{0}
$$


and positive invariance of $\mathcal{G}$

$$
(\boldsymbol{x}(t), \boldsymbol{z}(t)) \in \mathcal{G} \quad \forall t \in\left[t_{0}, \infty\right) .
$$

A control method that is able to fulfill both goals is called Invariance Control [7]; the region $\mathcal{G}$ is called an invariance region. Due to (5) subsystem (1)-(2) is called controlled dynamics and (3) internal dynamics.

It is well known that the above formulated semiglobal stability goal is not possible if system (1)-(3) is non-minimum phase [15]. In this case only local results can be achieved. Therefore we modify our control goal to: Find a large region $\mathcal{G} \subseteq \mathbb{R}^{n}$ and a controller $u(\boldsymbol{x}, \boldsymbol{z})$ such that with $(\boldsymbol{x}(0), \boldsymbol{z}(0)) \in \mathcal{G}$ the two goals (5) and (6) hold for controlled system (1)-(3). In this case standard linear control methods achieve small regions of attraction only. Existing nonlinear methods based on e.g. backstepping [4] or feedback passivation [12] do not apply for systems of the form (1)-(3). Therefore the main contribution of this paper is to solve the local stabilizing control problem with the invariance region $\mathcal{G}$ as a large region of attraction without the need of a control Lyapunov-function (clf) for the overall system (1)-(3).

\section{Invariance Control}

First necessary and sufficient conditions for a state space region to be positively invariant are given. We consider regions $\mathcal{G}$ defined implicitly by

$$
\mathcal{G}=\{(\boldsymbol{x}, \boldsymbol{z}) \mid \Phi(\boldsymbol{x}, \boldsymbol{z}) \leq 0\}
$$

where $\Phi \in \mathcal{C}^{1}$ is referred to as the invariance function [8]. The boundary of $\mathcal{G}$ is given by $\partial \mathcal{G}=\{(\boldsymbol{x}, \boldsymbol{z}) \mid \Phi(\boldsymbol{x}, \boldsymbol{z})=0\}$. With this representation a necessary and sufficient condition for $\mathcal{G}$ to be positively invariant is that the time derivative of $\Phi$ along the trajectory of system (1)-(3) must not increase on the boundary $\partial \mathcal{G}$, i.e.

$$
\dot{\Phi}=\nabla \Phi\left[\begin{array}{c}
x_{2} \\
0 \\
\boldsymbol{f}
\end{array}\right]+\nabla \Phi\left[\begin{array}{l}
0 \\
1 \\
\boldsymbol{g}
\end{array}\right] u \leq 0, \quad \forall(\boldsymbol{x}, \boldsymbol{z}) \in \partial \mathcal{G},
$$

where the $\nabla$-operator is used as the total derivative $d / d(\boldsymbol{x}, \boldsymbol{z})$, resulting in a row vector. In case $\nabla \Phi\left[\begin{array}{lll}0 & 1 & \boldsymbol{g}^{T}\end{array}\right]^{T} \neq 0$ we can always find appropriate values for $u \in \mathbb{R}$ so that the invariance condition (8) holds. Points where the control input vector field $\left[0 \begin{array}{ll}0 & \boldsymbol{g}^{T}\end{array}\right]^{T}$ of the overall system (1)-(3) lies in the tangent space of $\mathcal{G}$ and where in addition the term $\nabla \Phi\left[x_{2} 0 \boldsymbol{f}^{T}\right]^{T}$ is positive are called escape points [7] as there, no value of $u$ can keep the state $(\boldsymbol{x}, \boldsymbol{z})$ inside $\mathcal{G}$. Thus not every region is suitable to be controlled positively invariant.

In [8], design methods for Invariance Regions are presented for the case of a general $r$-time integrator subsystem. In these approaches, one has to find a function fulfilling a time decreasing condition along trajectories of a system of $n-11$ st order differential equations. A major simplification of the invariance region design process can be achieved when considering the case of relative degree two as in this case we only need a decreasing condition along the zero-dynamics of (1)-(3) w.r.t. $x_{1}=$ const, $x_{2}=0$. Beyond these savings of analytic computation the zero-dynamics are often already well examined in a preceding system analysis step, hence a first integral for the zero-dynamics, which is part of the invariance function, is often known in advance.

\subsection{Switching Linear Controller}

The linear subsystem (1)-(2) is controlled by the switched PDcontroller

$$
u\left(x_{1}^{*}(t), k_{d}(t), \boldsymbol{x}\right)=k_{p}\left(x_{1}^{*}(t)-x_{1}\right)-k_{d}(t) x_{2}
$$

with a piecewise constant switching velocity gain $k_{d}(t)>0$, a piecewise constant switching desired value $x_{1}^{*}(t)$, and a constant proportional gain $k_{p}>0$. The initial value for $k_{d}$ is denoted by $k_{d}^{i n i}>0$.

In this section we present a switching algorithm for $x_{1}^{*}(t)$ and $k_{d}(t)$ that achieves asymptotic stability for the linear subsystem (1)-(2). In Section 3.2 we show that the freedom of changing the transient behavior of the linear subsystem (1)-(2) by switching the control parameter $k_{d}(t)$ and the desired value $x_{1}^{*}(t)$ can be used to solve the invariance problem (8).

\begin{tabular}{|c|c|}
\hline Algorithm 1: ini & wait \\
\hline $\begin{array}{ll}\operatorname{act} 1: & x_{1}^{*}=x_{1}, k_{d}=k_{d}^{i n v} \\
\operatorname{act} 2: & k_{d}=k_{d}^{i n v} \\
\text { act3: } & x_{1}^{*}(t)=x_{1}, k_{d}=k_{d}^{i n i} \\
\operatorname{act} 4: & x_{1}^{*}=0, k_{d}=k_{d}^{i n i}\end{array}$ & $\begin{array}{l}\operatorname{tran} 1:(\boldsymbol{x}, \boldsymbol{z}) \in \partial \mathcal{G} \\
\operatorname{tran2:}(\boldsymbol{x}, \boldsymbol{z}) \in \partial \mathcal{G} \wedge x_{2}>0 \\
\operatorname{tran} 3: x_{2}=0 \\
\operatorname{tran} 4:(\boldsymbol{x}, \boldsymbol{z}) \in \chi\end{array}$ \\
\hline
\end{tabular}

The parameter switching algorithm for $x_{1}^{*}(t)$ and $k_{d}(t)$ is presented as a hybrid automaton with state transitions tran $i$ and actions act $i$. The basic functionality of Algorithm 1 is as fol-

lows: in the initial state of the controller ini the initial settings $x_{1}^{*}(t) \leq 0$ and $k_{d}^{i n i}$ are specified. In the controller state approach we have $x_{1}^{*}(t)=0, k_{d}=k_{d}^{i n i}$, hence the linear subsystem converges asymptotically towards the origin $\boldsymbol{x}=\mathbf{0}$. In the controller state wait, the damping of the linear subsystem is increased by switching $k_{d}(t)$ to a higher value $k_{d}^{i n v}$. Moreover $x_{1}^{*}(t)$ is set temporarily to the actual value of $x_{1}(t)$. Therefore after a short transient behavior to the new desired value $x_{1}^{*}(t)$ the convergence of the linear subsystem is stopped at the actual position $x_{1}^{*}$ and due to a special choice of $\Phi$ the internal state $\boldsymbol{z}$ runs along a stable trajectory $\left(x_{1}=x_{1}^{*}(t), \boldsymbol{z}(t)\right)$ of the zero dynamics until the transition condition tran 4 is fulfilled and the controller changes back into the state approach.

As will be explained in detail later on it is important for keeping $\mathcal{G}$ invariant to constrain the transient behavior of the controlled 
linear subsystem to aperiodic behavior. This is achieved by demanding

$$
k_{d}(t)^{2}-4 k_{p}>0
$$

for the switching parameter $k_{d}(t)$.

Therefore the linear subsystem (1)-(2) controlled by (9) has two real eigenvalues $\lambda_{1}, \lambda_{2}$ with a minimum eigenvalue $\lambda^{\text {min }}$. We now state a proposition on the transient behavior of the controlled linear subsystem, which is an important intermediate result for the following main theorem.

Proposition 1 Consider the linear subsystem (1)-(2) controlled by the switching linear controller (9). We assume without loss of generality $x_{1}(0) \leq 0$. If the initial state $\boldsymbol{x}(0)$ fulfills the condition

$$
x_{1}(0) \lambda^{\min } \geq x_{2}(0) \geq 0
$$

$k_{d}(t)$ and $x_{1}^{*}(t)$ are switched as determined by Algorithm 1 and $k_{d}(t)$ fulfills the aperiodic constraint (10), then $x_{2}(t) \geq 0$ for all times $t \geq 0$.

Proof: Without loss of generality we assume $\lambda_{1}<\lambda_{2}$. As both eigenvalues are real, we obtain by standard calculus the real solution $x_{2}(t)=c_{1} \lambda_{1} e^{\lambda_{1} t}+c_{2} \lambda_{2} e^{\lambda_{2} t}$ with two real constants $c_{1}=\left(-x_{2}(0)+x_{1}(0) \lambda_{2}\right) /\left(\lambda_{2}-\lambda_{1}\right)$ and $c_{2}=$ $\left(x_{2}(0)-x_{1}(0) \lambda_{1}\right) /\left(\lambda_{2}-\lambda_{1}\right)$.

State approach: The switching velocity gain is $k_{d}=k_{d}^{i n i}$. With constraint (11) for $x_{2}(0)$ we obtain $c_{2} \leq 0$ and $-c_{2} \geq c_{1}$. From this we can conclude $c_{1} \lambda_{1} \geq 0$ and hence $x_{2}(t) \geq 0$ in the case $c_{1} \leq 0$. If $c_{1}>0$ the relation $-c_{2} \lambda_{2} \leq c_{1} \lambda_{1}$ follows from $-c_{2}>c_{1}$, yielding $-c_{2} \lambda_{2} e^{\lambda_{2} t} \leq c_{1} \lambda_{1} e^{\lambda_{1} t}$ and hence $x_{2}(t) \geq 0$.

State wait: The switching desired value is $x_{1}^{*}(t)=x_{1}(t)$ and the switching velocity gain $k_{d}(t)$ is calculated so that (8) holds. If $x_{2}=0$ we again switch $x_{1}^{*}(t)=x_{1}(t)(\operatorname{tran} 3$, act3), resulting in $x_{1}(t)=$ const, $x_{2}(t)=0$ until the system changes back to the state approach. Thus we have $x_{2}(t) \geq 0$ during the active time of the state wait.

Assumption 2 There exists a $n$-dimensionally expanded open region $\chi \subseteq \mathcal{G}$ with the property that

i) every phase curve of all zero dynamics corresponding to an output function $h=x_{1}$ with ranging $x_{1} \in\left[x_{1}(0) ; 0\right]$ enters in finite time $\chi \backslash \partial \chi$,

ii) the subset $\chi \cap \partial \mathcal{G}$ is invariant for trajectories of system (1)(3) controlled by (9) with the initial setting of the switching parameters $k_{d}(t)=k_{d}^{i n i}$ and $x_{1}^{*}(t)=0$.

The above point $i$ ) can always be fulfilled since by Assumption 1 the zero-dynamics are at least locally stable. Point $i$ ) is needed to assure that the state $(\boldsymbol{x}, \boldsymbol{z})$ of system (1)-(3) with active controller state approach remains in $\mathcal{G} \backslash \partial \mathcal{G}$ for a finite time so that a dwell time argument assures asymptotic stability of the switched linear subsystem. It is remarkable that $\Phi$ represents a Lyapunov-function for the controlled overall system (1)-(3) in the region $\chi$. Assumption 2 completes the list of restrictive system assumptions together with Assumption 1. However these assumptions are still much easier to fulfill than global Lyapunov-function assumptions.

Proposition 2 Consider the linear subsystem (1)-(2) controlled by (9) with the switching parameters $k_{d}(t)$ and $x_{1}^{*}(t)$ determined by Algorithm 1 such that (10) holds. Under Assumption 1 and 2 the controlled linear subsystem is asymptotically stable.

Proof: Consider the quadratic positive definite Lyapunovlike function $V=1 / 2\left(p_{1} x_{1}^{2}+p_{2} x_{2}^{2}\right)$ with two constants $p_{1}>0, p_{2}>0$. The time derivative $\dot{V}$ along the trajectory $\boldsymbol{x}(t)$ of the linear subsystem (1)-(2) controlled by (9) while the controller state approach is active calculates as $\dot{V}=x_{1} x_{2}\left(p_{1}-p_{2} k_{p}\right)-p_{2} k_{d}^{i n i} x_{2}^{2}$. Choosing $p_{1}=p_{2} k_{p}$ yields $\dot{V}=-p_{2} k_{d}^{i n i} x_{2}^{2}$. We will next show that with each passing through the state wait, the function $V$ is non-increasing: Let $t_{i}$ denote the time when the state approach becomes passive and $t_{i+1}$ the time when it becomes active again. Due to Assumption 2 the time $t_{i+1}-t_{i}$ is finite. Both possible actions act 2 and act 3 which may occur during the passive time of state approach are so that the inequality conditions $0 \geq x_{1}\left(t \geq t_{i}\right) \geq x_{1}\left(t=t_{i}\right)$ and $0 \leq x_{2}\left(t \geq t_{i}\right) \leq x_{2}\left(t=t_{i}\right)$ hold, hence $V\left(t_{i+1}\right) \leq V\left(t_{i}\right)$. Therefore, with each passing through the state wait the Lyapunov-like function $V$ does not increase. Due to Assumption 2 the controller state approach remains active at least as long as the system state $(\boldsymbol{x}(t), \boldsymbol{z}(t))$ is located in the region $\chi$, guaranteeing a minimum time of stay within the controller state approach. Hence after each switching to approach a minimum time of convergence of the linear subsystem follows, assuring a minimum amount $\left|\Delta V^{\mathrm{min}}\right| \geq \gamma\|\boldsymbol{x}\|, \gamma>0$ of decrease of $V$ during the active time of approach. As shown e.g. in [6] this is enough argument to prove asymptotic stability of the switched linear subsystem.

\subsection{Main Theorem}

Consider the invariance function

$$
\Phi(\boldsymbol{x}, \boldsymbol{z})=\Phi_{0}\left(x_{1}, \boldsymbol{z}\right)+\gamma x_{2} .
$$

Assumption (1) assures that with the switching linear controller described in Section 3.1 for the linear subsystem (1)-(2) no escape points exist.

In order to compensate the influence of the control input $u$ on the internal dynamics (3) we calculate a positive $\gamma^{*}$ by the constraint optimization problem

$$
\begin{aligned}
& \gamma^{*}=\Theta \max _{\boldsymbol{x}, \boldsymbol{z}}\left|\frac{\partial \Phi_{0}\left(x_{1}, \boldsymbol{z}\right)}{\partial \boldsymbol{z}} \boldsymbol{g}(\boldsymbol{x}, \boldsymbol{z})\right| \\
& \text { w.r.t. }(i) \Phi_{0}\left(x_{1}, \boldsymbol{z}\right)+\gamma x_{2}=0 \\
& \qquad(i i) \gamma=\Theta\left|\frac{\partial \Phi_{0}\left(x_{1}, \boldsymbol{z}\right)}{\partial \boldsymbol{z}} \boldsymbol{g}(\boldsymbol{x}, \boldsymbol{z})\right|,
\end{aligned}
$$


with a constant $\Theta>1$. As $\Phi$ is radially unbounded by definition, there always exists a solution for $\gamma^{*}$.

Theorem 1 Consider system (1)-(3) controlled by the switched $P D$-controller (9) with velocity gain $k_{d}(t)$ and desired value $x_{1}^{*}(t)$ as piecewise constant switching parameters. The initial state is assumed to be located inside $\mathcal{G} \backslash \partial \mathcal{G}$. If $k_{d}(t)$ and $x_{1}^{*}(t)$ are determined by Algorithm 1, if (10) holds and if $x_{1}^{*}(t)$ and $k_{d}^{i n v}$ are chosen such that each time the trajectory $(\boldsymbol{x}(t), \boldsymbol{z}(t))$ hits $\partial \mathcal{G}$ the invariance condition

$$
\dot{\Phi}\left(\boldsymbol{x}, \boldsymbol{z}, k_{d}(t)=k_{d}^{i n v}, x_{1}=x_{1}^{*}(t)\right)<0, \forall(\boldsymbol{x}, \boldsymbol{z}) \in \partial \mathcal{G}
$$

is fulfilled, then

i) the state space region $\mathcal{G}$ is positively invariant

ii) the controlled linear subsystem is asymptotically stable.

Proof : $i)$ The time derivative $\dot{\Phi}$ along the trajectory of system (1)-(2) controlled by (9) follows with our choice of an invariance function (12) and with $\gamma^{*}$ from (13) to

$$
\dot{\Phi}=\frac{\partial \Phi_{0}}{\partial\left(x_{1}, \boldsymbol{z}\right)}\left[\begin{array}{c}
x_{2} \\
\boldsymbol{f}
\end{array}\right]+\left(\frac{\partial \Phi_{0}}{\partial \boldsymbol{z}} \boldsymbol{g}+\gamma^{*}\right) u
$$

with $u$ defined by (9). Due to (13) we have

$$
\frac{\partial \Phi_{0}}{\partial \boldsymbol{z}} \boldsymbol{g}+\gamma^{*}>0, \forall(\boldsymbol{x}, \boldsymbol{z}) \in \partial \mathcal{G}
$$

This and the fact that $x_{2} \geq 0$ due to Proposition 1 allows to conclude that whenever the system state hits the set $\partial \mathcal{G} \cap\left\{x_{2}>\right.$ $0\}$, there exists a suitable velocity gain $k_{d}^{i n v}(t)$ and $x_{1}^{*}(t)$ so that the invariance condition (8) holds.

ii) Asymptotic stability follows directly from Proposition 2.

Remark 1 It is possible to compute a set of parameters $\boldsymbol{k}^{*}=\left(k_{p}^{*}>0, k_{d}^{*}>0\right)$ such that

$$
\max _{(\boldsymbol{x}, \boldsymbol{z}) \in \partial \mathcal{G}} \dot{\Phi}\left(\boldsymbol{x}, \boldsymbol{z}, \boldsymbol{k}^{*}\right) \leq 0
$$

holds and $\mathcal{G}$ is positively invariant with constant $k_{p}=k_{p}^{*}$, $k_{d}=k_{d}^{*}$, i.e. without switching. However, the numerical computation of $\boldsymbol{k}$ may be difficult in case the optimization problem (15) is not convex. Moreover this constant gain approach may result in significantly higher gains than with the switching control law with the attendant problems of high energy consumption and additional stability problems in case of measurement noise, delay time or unmodelled dynamics.

\section{Experiments with R2D1}

The theory developed in the preceding sections is now applied to control the underactuated SCARA-type robot R2D1 shown in Fig. 1. The first joint is actuated by a direct-drive motor while the second joint is unactuated. The moving plane is inclined versus the gravitational field by an angle $\alpha=-\pi / 6$.

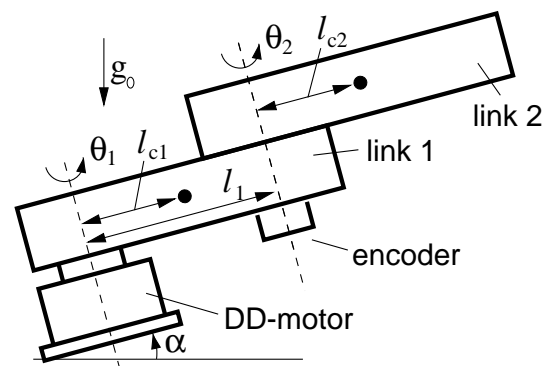

Figure 1: Schematic of R2D1.

The relevant physical parameters of R2D1 are estimated in standard units to $l_{1}=0.3, l_{c 1}=0.154, l_{c 2}=0.084$, $I_{1}=0.130, I_{2}=0.108, m_{1}=7.31, m_{2}=6.18, a_{1}=2.3$, $a_{2}=0.085, b_{1}=0.101$, and $b_{2}=0.005$. As there is one control input only, but two degrees of freedom, just one joint may be position controlled, while the second one remains uncontrolled and causes internal dynamics. Here, the control goal is to stabilize asymptotically the unactuated joint angle $\theta_{2}$ at a desired position $\theta_{2}^{d}$. Therefore an output function $y=\theta_{2}$ is defined with the desired value $y^{d}=\theta_{2}^{d}$. With $\theta_{2} \rightarrow \theta_{2}^{d}$ and $\dot{\theta_{2}} \rightarrow 0$ the internal dynamics approach the zero-dynamics, which mostly include unstable trajectories as shown in Fig. 2. Therefore R2D1 with $y=\theta_{2}$ belongs to the class of nonlinear non-minimum phase systems.

\subsection{Dynamics and Control Goal}

The dynamic equations of R2D1 are given by

$$
\boldsymbol{M}(\boldsymbol{\theta}) \ddot{\boldsymbol{\theta}}+\boldsymbol{n}(\boldsymbol{\theta}, \dot{\boldsymbol{\theta}})+\boldsymbol{g}^{*}(\boldsymbol{\theta})+\boldsymbol{r}(\boldsymbol{\theta})=\boldsymbol{\tau},
$$

with the joint angles $\boldsymbol{\theta}=\left[\begin{array}{ll}\theta_{1} & \theta_{2}\end{array}\right]^{T}$, the motor torque $\boldsymbol{\tau}=\left[\begin{array}{ll}\tau & 0\end{array}\right]^{T}$, the Coriolis and centrifugal torque $\boldsymbol{n}=\left[\begin{array}{ll}n_{1} & n_{2}\end{array}\right]^{T}$, the gravitational torque $\boldsymbol{g}^{*}=\left[\begin{array}{ll}g_{1}^{*} & g_{2}^{*}\end{array}\right]^{T}$, the inertia matrix $\boldsymbol{M}=\left[m_{i j}\right], i, j \in\{1,2\}$ and the frictional torque $\boldsymbol{r}=\left[\begin{array}{ll}r_{1} & r_{2}\end{array}\right]^{T}$. The dynamic parameters of (16) are

$$
\begin{aligned}
m_{11} & =A+2 B \cos \theta_{2} & & n_{1}=-B\left(\dot{\theta}_{2}^{2}+2 \dot{\theta}_{1} \dot{\theta}_{2}\right) \sin \theta_{2} \\
m_{12} & =m_{22}+B \cos \theta_{2} & n_{2} & =B \dot{\theta}_{1}^{2} \sin \theta_{2} \\
m_{22} & =m_{2} l_{c 2}^{2}+I_{2} & g_{1}^{*} & =g_{2}-C \sin \alpha \sin \theta_{1} \\
r_{i} & =a_{i} \operatorname{sign}\left[\dot{\theta}_{i}\right]+b_{i} \dot{\theta}_{i} & & g_{2}^{*}=-D \sin \alpha \sin \left(\theta_{1}+\theta_{2}\right),
\end{aligned}
$$

with sign $[0]=0$ and $A=m_{1} l_{c 1}^{2}+m_{2}\left(l_{1}^{2}+l_{c 2}^{2}\right)+I_{1}+I_{2}$, $B=m_{2} l_{1} l_{c 2}, C=g_{0}\left(m_{1} l_{c 1}+m_{2} l_{1}\right), D=g_{0} m_{2} l_{c 2}$. Solv-

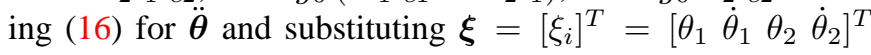
transforms (16) into the standard SISO-form

$$
\dot{\boldsymbol{\xi}}=\hat{\boldsymbol{f}}(\boldsymbol{\xi})+\hat{\boldsymbol{g}}(\boldsymbol{\xi}) \tau
$$

with drift $\hat{\boldsymbol{f}}=\left[\hat{f}_{i}\right]$ and control input vector field $\hat{\boldsymbol{g}}=\left[\hat{g}_{i}\right], i \in$ $\{1, \ldots, 4\}$.

The output function is defined as $h(\boldsymbol{\xi})=\xi_{3}$ with the desired value $\xi_{3}^{d}=\theta_{2}^{d}$. In order to feedback linearize system (17) to

\footnotetext{
${ }^{1}$ Note that the gravitational torque is marked by an asterisk * to distinguish
} it from the control input vector field $\boldsymbol{g}(\boldsymbol{x}, \boldsymbol{z})$. 
normal form, the invertible coordinate transformation $(\boldsymbol{x}, \boldsymbol{z})=$ $\boldsymbol{\Psi}(\boldsymbol{\xi})$ and its inverse $\boldsymbol{\xi}=\boldsymbol{\Psi}^{-1}(\boldsymbol{x}, \boldsymbol{z})$ with

$$
\boldsymbol{\Psi}=\left[\begin{array}{c}
\xi_{3} \\
\xi_{4} \\
\xi_{1} \\
\xi_{2}-\xi_{4} \hat{g}_{2} / \hat{g}_{4}\left(\xi_{3}\right)
\end{array}\right], \boldsymbol{\Psi}^{-1}=\left[\begin{array}{c}
z_{1} \\
z_{2}+x_{2} \hat{g}_{2} / \hat{g}_{4}\left(x_{1}\right) \\
x_{1} \\
x_{2}
\end{array}\right]
$$

is used to transform (17) to a global normal form. Applying the state feedback linearizing control law $\tau=(u-b(\boldsymbol{\xi})) a(\boldsymbol{\xi})^{-1}$ with $a(\boldsymbol{\xi})=\mathrm{L}_{\hat{\boldsymbol{g}}} \mathrm{L}_{\hat{\boldsymbol{f}}} h(\boldsymbol{\xi})$ and $b(\boldsymbol{\xi})=\mathrm{L}_{\hat{\boldsymbol{f}}}^{2} h(\boldsymbol{\xi})$, we obtain the cascade

$$
\begin{aligned}
\dot{x}_{1} & =x_{2} \\
\dot{x}_{2} & =u \\
\dot{z}_{1} & =q_{3}(\boldsymbol{x}, \boldsymbol{z}) \\
\dot{z}_{2} & =q_{4}(\boldsymbol{x}, \boldsymbol{z})
\end{aligned}
$$

with $q_{i}(\boldsymbol{x}, \boldsymbol{z})=\left.\mathrm{L}_{\hat{\boldsymbol{f}}} \Psi_{i}\right|_{\boldsymbol{\Psi}^{-1}(\boldsymbol{x}, \boldsymbol{z})}, i \in\{3,4\}$.

The above feedback linearization is valid only if strong inertial coupling holds [13], i.e. $m_{12} \neq 0$. With the present system configuration this yields $\left|\theta_{2}\right|<166^{\circ}$.

Note that the normal form coordinates $z$ still all have a meaningful physical interpretation since $z_{1}=\xi_{3}=\theta_{2}$ and $z_{2}=\dot{\theta}_{1}-\dot{\theta}_{2} \hat{g}_{2} / \hat{g}_{4}\left(\theta_{2}\right)$ represents the momentum acting on the second joint divided by the term $m_{12}$.

The coordinate transformation (1) is such that $\mathrm{L}_{\hat{\boldsymbol{g}}} \Psi_{i}=0$, $i \in\{3,4\}$. Therefore the control input $u$ does not affect the internal dynamics in normal form coordinates $(\boldsymbol{x}, \boldsymbol{z})$, i.e. (18)(21) is in cascaded form. As a consequence the optimization (13) is not necessary anymore as $\gamma$ may be set to any positive number. See also related work by [9] for normal forms of underactuated mechanical systems.

\subsection{Invariance Region Design}

As shown in Fig. 2 there exist stable and unstable phase-curves of the zero-dynamics separated by a homoclinic orbit. We obtain a suitable function $\Phi_{0}$ by the implicit solution for the phase curves of the zero-dynamics of R2D 1 with $\mu \in(0 ; \pi)$ as a free parameter measuring the distance between the unstable equilibrium point $P_{u}$ and the stable phase curve. See Fig. 2 (a) for two examples of phase curves with two different values $\mu_{1}$ and $\mu_{2}$. For constant $x_{1}=x_{1}^{*}(t)$ we choose the boundary $\partial \mathcal{G}_{0}$ such that it coincides with a stable phase curve of the zerodynamics. The whole boundary $\partial \mathcal{G}_{0}$ is put together from stable phase curves for a constant $\mu$ and $x_{2}=0$, see also Fig. 2 (b) for a 3D-vision of $\partial \mathcal{G}_{0}$. Numerical calculations have shown, that a suitable region $\chi$ fulfilling (2) is the surrounding of the line between the stable and the unstable equilibrium point $P_{s}$ and $P_{u}$, i.e. the surrounding of $\left\{\left(z_{2}=0\right) \wedge\left(-x_{1}-\pi<z_{1}<-x_{1}\right)\right\}$.

\subsection{Discussion of Experimental Results}

The controller gains were set to $k_{p}=180, k_{d}^{i n i}=2 k_{p}^{1 / 2}+1$. The sampling time was $\Delta T=1 \mathrm{~ms}$. Parameters determining

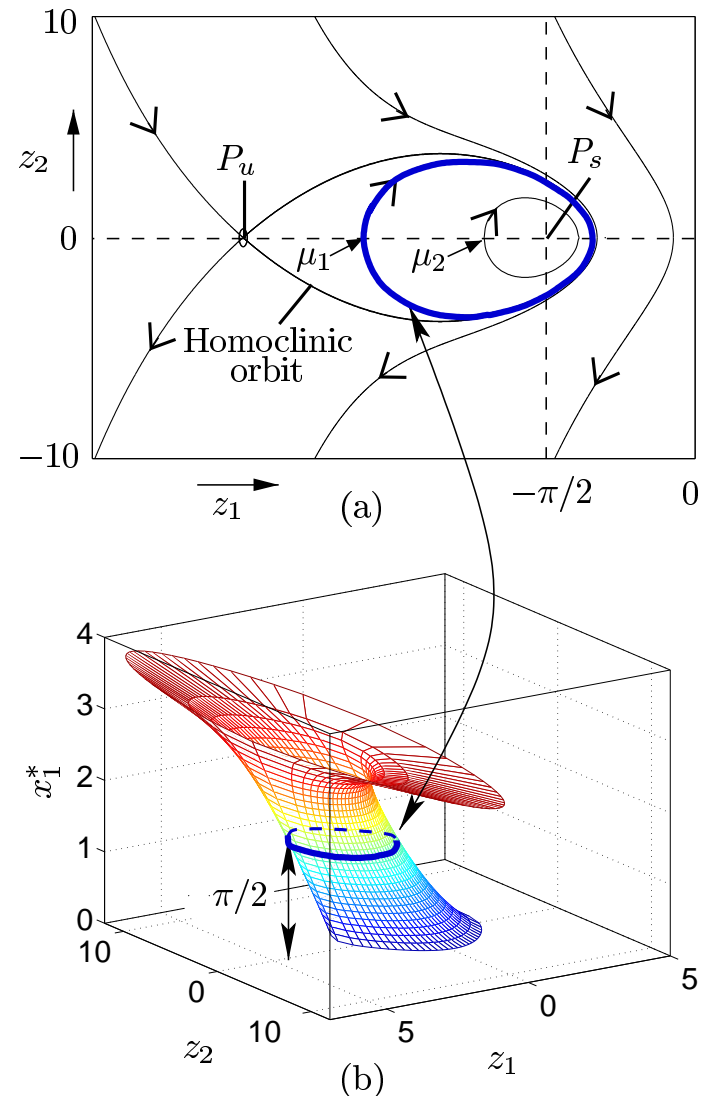

Figure 2: (a) Phase plot of zero-dynamics for $x_{1}=\pi / 2$. (b) Boundary $\partial \mathcal{G}_{0}$ for $\mu=\mu_{1}=1$.

the shape of the invariance region were chosen as $\mu=1$ and $|\gamma|=0.5$. The sign of the desired value is toggled between $\pm 75^{\circ}$ (see the red line in Fig. 3).

Note that if $x_{2}<0$ due to the negative desired value $\theta_{2}^{d}<0$ the parameter $\gamma$ must also be negative. As can be seen in Fig. 3 (a) the controlled state $\boldsymbol{x}$ converges asymptotically towards the desired value while the internal dynamics $z$ remain stable. As shown in Fig. 3 (b), the switching Invariance Controller keeps the region $\mathcal{G}$ invariant on all points. This is the merit of switching $x_{1}^{*}(t)$ and $k_{d}(t)$ as shown in the last two plots of Fig. 3 (b). When the system trajectory hits the boundary of $\mathcal{G}$, i.e. $\Phi=0$, the parameters $x_{1}^{*}(t)$ and $k_{d}(t)$ are switched such that $\dot{\Phi}<-20$ and the trajectory moves back into the interior of $\mathcal{G}$. This can be seen at the point marked with (1) in Fig. 3 (b). As shown in [11] robustness with respect to uncertain physical parameters can be achieved by demanding $\dot{\Phi}<-\epsilon$ with $\epsilon>0$ instead of $\dot{\Phi} \leq 0$, where the size of $\epsilon$ depends upon the amount of perturbations. Moreover the method can easily be made robust w.r.t. time discrete implementation by introducing a distance to the boundary $\partial \mathcal{G}$ such that the invariance control detects the state-entering into a small boundary-layer, i.e. $\Phi \geq-0.1$ instead of $\Phi=0$. See also http://www. invariancecontrol. de for a movie of this experiment and further information on Invariance-Control. 

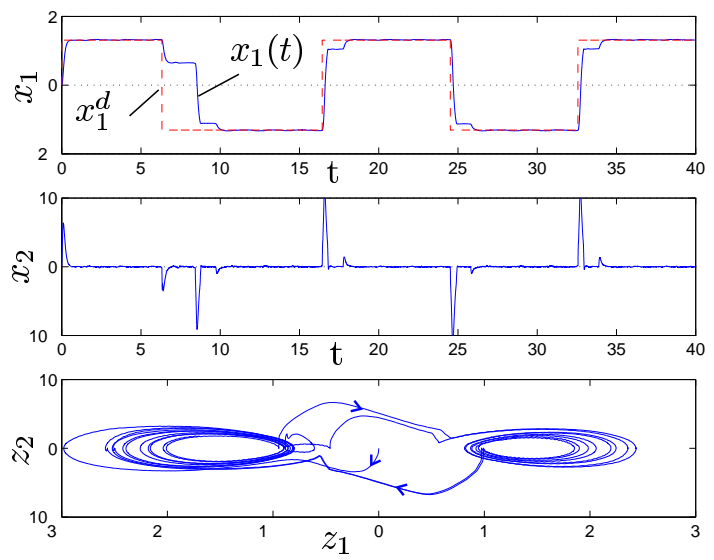

(1)
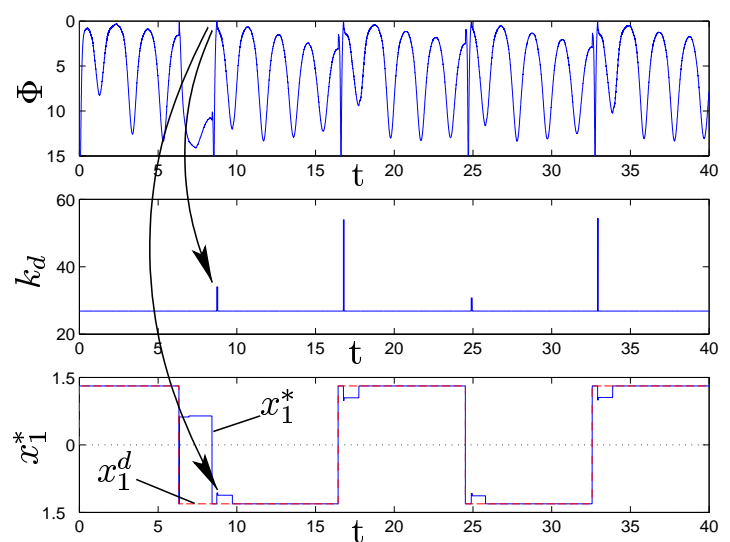

(b)

Figure 3: (a) Invariance controlled system states $\boldsymbol{x}$ and phase plot of the bounded internal dynamics. (b) Effect of switching $k_{d}(t)$ and $x_{1}^{*}(t)$ on the invariance function $\Phi$.

\section{Conclusions and Outlook}

The main result of this paper is an intelligent switching strategy for control parameters and an invariance region design method which requires only the analytic knowledge of phase curves of the zero-dynamics. This is a major simplification compared with the Invariance Control design method for more general classes of systems, as the zero-dynamics are often well known from a preceding system analyzing step. Thus for a large class of underactuated systems with relative degree two, Invariance Control can be applied. The invariance region forms together with the switching strategy an intelligent switching control which is able to trade off between convergence of the controlled linear subsystem and invariance of a region. The proposed theory has shown its applicability and robustness in an underactuated robot experiment. Furthermore it can be shown that robustness of invariance w.r.t. limited control input can be achieved by changing the geometric shape design parameters $\mu$ and $\gamma$.

Future work is to address the question if the assumption of a region $\chi$ for the system under consideration is necessary for the system to be stabilizable at all.

\section{References}

[1] S. A. Al-Hiddabi and N. H. McClamroch, "Trajectory Tracking Control and Maneuver Regulation Control for the CTOL Aircraft Model," in Proceedings of the IEEE Conference on Decision and Control, (Phoenix, Arizona), pp. 1958-1963, 1999. 1

[2] A. M. Bloch and N. E. Leonard, "Stabilization of Mechanical Systems Using Controlled Lagrangians," in Proceedings of the IEEE Conference on Decision and Control, (San Diego), pp. 2356-2361, 1997. 1

[3] R. M. Hirschorn and E. Aranda Bricaire, "Practical stabilization of locally linearizable systems," in Proceedings of the IEEE Conference on Decision and Control, (Phoenix, Arizona), pp. 1623-1628, 1999. 1

[4] M. Krstić, I. Kanellakopoulos, and P. Kokotović, Nonlinear and Adaptive Control Design. John Wiley \& Sons, Inc., 1995. 1, 2

[5] S. M. Lee, N. H. Jo, and J. H. Seo, "Feedforward Stabilization of Nonlinear Nonminimum Phase Systems," in Proceedings of the IEEE Conference on Decision and Control, (Phoenix, Arizona), pp. 1284-1289, 1999. 1

[6] D. Liberzon and A. S. Morse, "Basic Problems in Stability and Design of Switched Systems," IEEE Control Systems Magazine, vol. 19, pp. 59-70, 10 1999. 3

[7] J. Mareczek and M. Buss, "Preliminary Studies on Geometric Invariance Control Synthesis," in Proceedings of European Control Conference, (Karlsruhe, Germany), 1999. 1, 2

[8] J. Mareczek, M. Buss, and G. Schmidt, "Sufficient Conditions for Invariance Control of a Class of Nonlinear Systems," in Proceedings of the 39th IEEE Conference on Decision and Control, (Sydney, Australia), December 2000. 1, 2

[9] R. Olfati Saber and A. Megretski, "Controller design for a class of underactuated nonlinear Systems," in Proceedings of the IEEE Conference on Decision and Control, (Tampa, FL), pp. 4182-4187, 1998. 5

[10] M. Reyhanoglu, A. van der Schaft, N. H. McClamroch, and I. Kolmanovsky, "Dynamics and Control of a Class of Underactuated Mechanical Systems," IEEE Transactions on Automatic Control, vol. 44, pp. 1663-1671, September 1999. 1

[11] C. Schweiger and J. Mareczek, "Robust Switching Invariance Control of an Underactuated Robot Experiment," in Dynamics, Bifurcations and Control (M. Thoma, ed.), Lecture Notes in Control and Information Sciences, Springer-Verlag, submitted for review. 5

[12] R. Sepulchre, M. Janković, and P. Kokotović, Constructive Nonlinear Control. London: Springer-Verlag, 1 ed., 1997. 2

[13] M. W. Spong, "Partial Feedback Linearization of Underactuated Mechanical Systems," in Proceedings of the IEEE/RSJ/GI International Conference on Intelligent Robots and Systems IROS, (München), pp. 314-321, 1994. 5

[14] M. W. Spong, "Energy Based Control of a Class of Underactuated Mechanical Systems," in 13th IFAC Triennial World Congress, (San Francisco), pp. 431-435, 1996. 1

[15] H. J. Sussmann, "Limitations on the Stabilizability of Globally Minimum Phase Systems," IEEE Transactions on Automatic Control, vol. 35, pp. 117-119, January 1990. 2 